\title{
Disclosure of interests
}

This book only contains the views of the author and not those of its funding organizations. This is especially important to emphasize in a book resulting from research projects partly financed by one actor in the domain name industry (the Norwegian country-code TLD operator UNINETT Norid AS). The author has not received any content-related instructions from the funding organizations. Nevertheless, the risk of bias due to economic incentives is acknowledged. I have attempted to manage this risk primarily by limiting the scope of the book to gTLD issues, to the exclusion of ccTLDs. However, this is only a partial solution because gTLDs can be perceived to compete partly with ccTLDs. Therefore, the book also contains more limited discussions of regulatory issues that affect ccTLD competition with gTLDs, such as in the context of geographic gTLDs - for example, those including country names. 